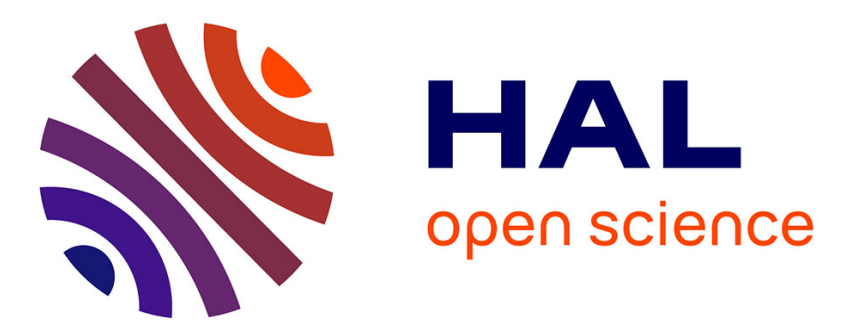

\title{
A note on distributed finite-time observers
}

Haik Silm, Rosane Ushirobira, Denis Efimov, Jean-Pierre Richard, Wim Michiels

\section{To cite this version:}

Haik Silm, Rosane Ushirobira, Denis Efimov, Jean-Pierre Richard, Wim Michiels. A note on distributed finite-time observers. IEEE Transactions on Automatic Control, 2018, 10.1109/TAC.2018.2838042 . hal-01827041v2

\section{HAL Id: hal-01827041 https://hal.inria.fr/hal-01827041v2}

Submitted on 7 Nov 2019

HAL is a multi-disciplinary open access archive for the deposit and dissemination of scientific research documents, whether they are published or not. The documents may come from teaching and research institutions in France or abroad, or from public or private research centers.
L'archive ouverte pluridisciplinaire HAL, est destinée au dépôt et à la diffusion de documents scientifiques de niveau recherche, publiés ou non, émanant des établissements d'enseignement et de recherche français ou étrangers, des laboratoires publics ou privés. 


\title{
A note on distributed finite-time observers
}

\author{
Haik Silm, Rosane Ushirobira, Denis Efimov, Jean-Pierre Richard, Wim Michiels
}

\begin{abstract}
The robust distributed estimation for a class of time-invariant plants is achieved via a finite-time observer, its error reaching zero after a finite time in the absence of perturbation. Two types of robustness are also shown. First, input-to-state stability with respect to measurement noises and additive perturbations is proven. Second, we demonstrate that the estimation error stays bounded in the presence of known transmission delays.
\end{abstract}

Index Terms-Distributed estimation problem, finite-time observers, weighted homogeneity, time-delay.

\section{INTRODUCTION}

Recent developments in technology have enabled to employ more and more distributed networked systems, where a large set of agents endowed with computational capacities communicate with each other to perform collaborative tasks. In control theory, this has created an increasing demand for decentralized solutions of previously solely centralized concepts. The communication network between the agents comes here explicitly into play, formally described by a graph which is dictated by the neighborhood relations of the agents. For state observation, this has given rise to the distributed estimation problem [1]. In this setting each agent measures a different output of a (large-scale) system. The goal is to design a distributed observer, such that all agents are able to reconstruct the full state of the system using only their individual measurement and state estimates from neighboring agents, while the challenge is that not a single agent is able to do so using only its measurement.

Up to now the solvability of the distributed estimation problem under a given graph has been established among others in [2]-[4], which loosely speaking amounts to connectivity conditions on the communication graph. Furthermore, different designs of distributed observers for discrete and continuous-time linear systems have been proposed based on Luenberger Observers [5], Kalman Filtering [6] or Moving Horizon Estimation [7], to mention a few. While they vary in their aims, as for example being robust with respect to disturbances as in [8], a key characteristic of an observer is the convergence rate of the error. This has only recently been addressed in [9], where a design was proposed yielding a

H. Silm and J.-P. Richard are with Univ. Lille, Inria, CNRS, Centrale Lille, UMR 9189 - CRIStAL - Centre de recherche en Informatique Signal et Automatique de Lille, F-59000 Lille, France (e-mail: haik.silm@inria.fr; jean-pierre.richard@centralelille.fr).

R. Ushirobira and D. Efimov are with Inria, Univ. Lille, CNRS, UMR 9189 - CRIStAL - Centre de Recherche en Informatique Signal et Automatique de Lille, F-59000 Lille, France (e-mail: rosane.ushirobira@inria.fr; denis.efimov@inria.fr).

H. Silm and W. Michiels are with the Department of Computer Science, KU Leuven, Celestijnenlaan 200A, 3001 Heverlee, Belgium (e-mail: wim.michiels@cs.kuleuven.be). linear distributed observer capable of estimating the state at an arbitrary asymptotic convergence rate.

The contribution of this work is to propose an observer for the distributed estimation problem, which reaches the correct estimate in an ideal noise-free case in finite time instead of asymptotically. In addition, we will show that such a nonlinear observer possesses interesting robustness features with respect to external disturbances or noises and communication delays, which are natural issues for large-scale and distributed systems. This follows the recent achievements of developing finite-time estimators $[10]-[14]$ in the centralized setting. This work is inspired by the results of [15] and [12], where an observer has been designed whose error dynamics is homogeneous with negative degree. We apply that approach to the distributed estimation of a system composed of multiple chain of integrators (a preliminary version of this, without proofs, was presented in [16]) and by robustness arguments the result is extended to more general classes of plants with communication delays. A proposal of a fixed-time converging distributed observer is also briefly presented.

The outline of this technical note is as follows. First, underlying stability concepts and the notion of weighted homogeneity are presented in Section III. The main steps of [12] are recapitulated in Section III with a small extension (stating an explicit bound for the degree of homogeneity). In Section IV the distributed finite-time converging observer is presented, and a discussion on its robustness with respect to disturbances, measurement noise and transmission delays follows. A simulation example is presented in Section V. Concluding remarks and future works are discussed in Section VI

\section{Notation}

- For $x=\left[\begin{array}{lll}x_{1} & \ldots & x_{n}\end{array}\right]^{\mathrm{T}} \in \mathbb{R}^{n}$ and $\alpha=\left[\begin{array}{lll}\alpha_{1} & \ldots & \alpha_{n}\end{array}\right]^{\mathrm{T}} \in \mathbb{R}_{+}^{n}$, we denote by $\lceil x\rfloor^{\alpha}=\left[\begin{array}{lll}\operatorname{sign}\left(x_{1}\right)\left|x_{1}\right|^{\alpha_{1}} & \ldots & \operatorname{sign}\left(x_{n}\right)\left|x_{n}\right|^{\alpha_{n}}\end{array}\right]^{\mathrm{T}}$ the sign preserving element-wise exponentiation.

- $\operatorname{diag}(x)$ denotes the diagonal matrix with $x_{1}, \ldots, x_{n}$ on the main diagonal and 0 otherwise, and $\operatorname{diag}\left\{B_{i}\right\}_{i=1}^{N}$ is a $N p \times$ $N q$ block diagonal matrix with blocks $B_{1}, \ldots, B_{N} \in \mathbb{R}^{p \times q}$.

- $1_{n} \in \mathbb{R}^{n}$ is the vector with all entries equal $1, I_{n}$ is the $n \times n$ identity matrix.

- $\lambda_{\min }(P)$ and $\lambda_{\max }(P)$ are the smallest and the greatest eigenvalues of a real symmetric matrix $P$, respectively.

- A sequence of integers $1,2, \ldots, n$ is denoted by $\overline{1, n}$.

- For a closed set $S \subset \mathbb{R}^{n}$, denote its boundary as $\partial S$.

- $\|\cdot\|$ denotes the Euclidean norm on $\mathbb{R}^{n}$.

- $\mathcal{L}_{\infty}$ is the set of essentially bounded measurable functions $\mathbb{R} \rightarrow \mathbb{R}^{m}$ with the norm $\|\cdot\|_{[0,+\infty)}=\sup _{t \in[0,+\infty)}\|\cdot\|$. 


\section{PRELIMINARIES}

Consider a generic nonlinear system:

$$
\dot{x}(t)=f(x(t), d(t)), t \geq 0,
$$

where $x \in \mathbb{R}^{n}$ is the state, $d \in \mathbb{R}^{m}$ is the input, $f: \mathbb{R}^{n} \times \mathbb{R}^{m} \rightarrow$ $\mathbb{R}^{n}$ ensures forward existence and uniqueness of the system solutions (at least locally) for $d \in \mathcal{L}_{\infty}$ and $f(0,0)=0$. For an initial condition $x_{0} \in \mathbb{R}^{n}$ and an input $d$, the corresponding solution is $X\left(t, x_{0}, d\right), \forall t \geq 0$ for which the solution exists.

Following [17]-[19], let $\Omega$ be an open neighborhood of the origin in $\mathbb{R}^{n}$.

Definition 1: At the steady state $x=0$ the system (1) with $d=0$ is said to be

(a) stable on $\Omega$ if for any $x_{0} \in \Omega, X\left(t, x_{0}, 0\right)$ is defined for all $t \geq 0$, and for any $\varepsilon>0$ there is $\delta>0$ such that for any $x_{0} \in \Omega$, if $\left\|x_{0}\right\| \leq \delta$ then $\left\|X\left(t, x_{0}, 0\right)\right\| \leq \varepsilon, \forall t \geq 0$;

(b) asymptotically stable on $\Omega$ if it is stable and for any $\kappa>0$ and $\varepsilon>0$ there exists $T(\kappa, \varepsilon) \geq 0$ such that for any $x_{0} \in$ $\Omega$, if $\left\|x_{0}\right\| \leq \kappa$ then $\left\|X\left(t, x_{0}, 0\right)\right\| \leq \varepsilon$ for all $t \geq T(\kappa, \varepsilon)$;

(c) finite-time stable on $\Omega$ if it is stable and finite-time converging from $\Omega$, i.e. for any $x_{0} \in \Omega$ there exists $0 \leq T<+\infty$ such that $X\left(t, x_{0}, 0\right)=0$ for all $t \geq T$. The function $T_{0}\left(x_{0}\right)=\inf \left\{T \geq 0: X\left(t, x_{0}, 0\right)=0, \forall t \geq T\right\}$ is called the settling time of the system (1);

(d) fixed-time stable on $\Omega$ if it is finite-time stable and $\sup _{x_{0} \in \Omega} T_{0}\left(x_{0}\right)<+\infty$.

The set $\Omega$ is called the domain of stability/attraction.

If $\Omega=\mathbb{R}^{n}$, then the corresponding properties are called global stability/asymptotic stability/finite-time/fixed-time stability of (1) at $x=0$. Similarly, these stability notions can be defined with respect to a compact invariant set, by replacing the distance to the origin in Definition 1 with the distance to the invariant set.

In the case of an input $d \neq 0$ we are interested in the following stability property [20]:

Definition 2: The system [1] is called input-to-state stable (ISS), if for any input $d \in \mathcal{L}_{\infty}$ and any $x_{0} \in \mathbb{R}^{n}$ there are some functions $\beta \in \mathcal{K} \mathcal{L}, \gamma \in \mathcal{K}$ such that for all $t \geq 0$

$$
\left\|X\left(t, x_{0}, d\right)\right\| \leq \beta\left(\left\|x_{0}\right\|, t\right)+\gamma\left(\|d\|_{[0, t)}\right) .
$$

For the definition of classes of functions $\mathcal{K}, \mathcal{K}_{\infty}$ and $\mathcal{K} \mathcal{L}$, we refer to [18].

\section{A. Weighted homogeneity}

Following [21], [22], for strictly positive numbers $r_{i}, i=\overline{1, n}$ called weights and $\lambda>0$, define:

- the vector of weights $\mathbf{r}=\left[\begin{array}{lll}r_{1} & \ldots & r_{n}\end{array}\right]^{T}, r_{\max }=$ $\max _{1 \leq j \leq n} r_{j}$ and $r_{\min }=\min _{1 \leq j \leq n} r_{j}$

- the dilation matrix function $\Lambda_{r}(\lambda)=\operatorname{diag}\left\{\lambda^{r_{i}}\right\}_{i=1}^{n}$. Note that $\forall x \in \mathbb{R}^{n}$ and $\forall \lambda>0$ we have $\Lambda_{r}(\lambda) x=$ $\left[\begin{array}{lll}\lambda^{r_{1}} x_{1} & \ldots & \lambda^{r_{n}} x_{n}\end{array}\right]^{T}$;

- the $\mathbf{r}$-homogeneous norm $\|x\|_{r}=\left(\sum_{i=1}^{n}\left|x_{i}\right|^{\frac{\rho}{r_{i}}}\right)^{\frac{1}{\rho}}$ for any $x \in \mathbb{R}^{n}$ and $\rho \geq r_{\max }$. This is not a norm in the standard sense, since the triangle inequality is not satisfied for $\|\cdot\|_{r}$, however there exist $\underline{\sigma}, \bar{\sigma} \in \mathcal{K}_{\infty}$ such that

$$
\underline{\sigma}\left(\|x\|_{r}\right) \leq\|x\| \leq \bar{\sigma}\left(\|x\|_{r}\right) \quad \forall x \in \mathbb{R}^{n} ;
$$

- for $\rho \geq 0$, the sphere and the ball in the homogeneous norm $S_{r}(\rho)=\left\{x \in \mathbb{R}^{n}:\|x\|_{r}=\rho\right\}$ and $B_{r}(\rho)=\left\{x \in \mathbb{R}^{n}\right.$ : $\left.\|x\|_{r} \leq \rho\right\}$.

Definition 3: A function $g: \mathbb{R}^{n} \rightarrow \mathbb{R}$ is $\mathbf{r}$-homogeneous with degree $\mu \in \mathbb{R}$ if $\forall x \in \mathbb{R}^{n}$ and $\forall \lambda>0$ we have $\lambda^{-\mu} g\left(\Lambda_{r}(\lambda) x\right)=$ $g(x)$. A vector field $f: \mathbb{R}^{n} \rightarrow \mathbb{R}^{n}$ is $\mathbf{r}$-homogeneous with degree $v \in \mathbb{R}$, with $v \geq-r_{\min }$ if $\forall x \in \mathbb{R}^{n}$ and $\forall \lambda>0$ we have $\lambda^{-v} \Lambda_{r}^{-1}(\lambda) f\left(\Lambda_{r}(\bar{\lambda}) x\right)=f(x)$ (this means that the $i^{\text {th }}$ component of $f$ is a $\mathbf{r}$-homogeneous function of degree $r_{i}+v$ ).

The system (1) with $d=0$ is $\mathbf{r}$-homogeneous of degree $v$ if the vector field $f$ is $\mathbf{r}$-homogeneous of the degree $v$.

Theorem 1 ([21], [23], [24]): For the system (1) with $d=0$ and $\mathbf{r}$-homogeneous and continuous function $f$ the following properties are equivalent:

- the system (1) is (locally) asymptotically stable;

- there exists a continuously differentiable $\mathbf{r}$-homogeneous Lyapunov function $V: \mathbb{R}^{n} \rightarrow \mathbb{R}_{+}$such that

$$
\begin{gathered}
\alpha_{1}(\|x\|) \leq V(x) \leq \alpha_{2}(\|x\|), \\
L_{f} V(x)=\frac{\partial V}{\partial x}(x) f(x) \leq-\alpha(\|x\|), \\
\lambda^{-\mu} V\left(\Lambda_{r}(\lambda) x\right)=V(x), \mu>r_{\max },
\end{gathered}
$$

$\forall x \in \mathbb{R}^{n}$ and $\forall \lambda>0$, for some $\alpha_{1}, \alpha_{2} \in \mathcal{K}_{\infty}$ and $\alpha \in \mathcal{K}$;

- there is a compact strictly positively invariant set $S$ containing the origin (i.e. $X\left(t, x_{0}, 0\right) \in S \backslash \partial S$ for all $t>0$ and all $x_{0} \in \partial S$ ).

The requirement on continuity of the function $f$ has been relaxed in [25] (the function $V$ can still be selected smooth).

Theorem 2 ([26]): If (1) with $d=0$ is $\mathbf{r}$-homogeneous of degree $v$ and asymptotically stable at the origin, then it is

(i) globally finite-time stable at the origin if $v<0$;

(ii) globally exponentially stable at the origin if $v=0$;

(iii) globally fixed-time stable with respect to the unit ball $B_{r}(1)$, if $v>0$.

For $d \neq 0$ in 1 , let $\tilde{f} \in \mathbb{R}^{n+m}$ be an auxiliary vector field, defined as $\tilde{f}=\left[\begin{array}{l}f \\ 0\end{array}\right]$.

Theorem 3 ([27]): Let $\tilde{f}$ be homogeneous with the weights $\mathbf{r}=\left[\begin{array}{lll}r_{1} & \ldots & r_{n}\end{array}\right]>0, \tilde{\mathbf{r}}=\left[\begin{array}{lll}\tilde{r}_{1} & \ldots & \tilde{r}_{m}\end{array}\right]>0$ with a degree $v \geq-r_{\min }$, i.e. $f\left(\Lambda_{r}(\lambda) x, \Lambda_{\tilde{r}}(\lambda) d\right)=\lambda^{v} \Lambda_{r}(\lambda) f(x, d)$ for all $x \in \mathbb{R}^{n}, d \in \mathbb{R}^{m}$ and all $\lambda>0$. Assume that the system (1) is globally asymptotically stable for $d=0$, then (1) is ISS.

\section{FINITE-TIME OBSERVER DESIGN}

To introduce later a distributed finite-time observer, let us briefly recall a centralized concept of finite-time estimator from [12]. For this purpose consider a chain of integrators:

$$
\dot{x}=A x, \quad y=c^{\mathrm{T}} x,
$$

where $x \in \mathbb{R}^{n}$ is the state, $y \in \mathbb{R}$ is the output of the system and the matrix $A \in \mathbb{R}^{n \times n}$ and the output vector $c \in \mathbb{R}^{n}$ are:

$$
A=\left[\begin{array}{ccccc}
0 & 1 & 0 & \ldots & 0 \\
0 & 0 & 1 & \ldots & 0 \\
& & \ddots & & \\
0 & 0 & \ldots & 0 & 1 \\
0 & 0 & \ldots & 0 & 0
\end{array}\right], \quad c=\left[\begin{array}{c}
1 \\
0 \\
\vdots \\
0
\end{array}\right] .
$$


Note that any fully observable linear system can be transformed into a chain of integrators modulo an output injection, as well as some nonlinear systems [28], [29].

Based on Luenberger observer design, a finite-time observer has been constructed in [12] as

$$
\dot{z}=A z+\operatorname{diag}(\ell)\left[\left(c^{\mathrm{T}} z-y\right) 1_{n}\right\rfloor^{\gamma},
$$

where $z \in \mathbb{R}^{n}$ is the estimate of the state $x$, the observer gain $\ell \in \mathbb{R}^{n}$ and $\gamma \in \mathbb{R}^{n}$ are defined later. Introducing the observer error as $e=z-x$ yields with (2) and (3) the error system:

$$
\dot{e}=f(e)=A e+\operatorname{diag}(\ell)\left[\left(c^{\mathrm{T}} e\right) 1_{n}\right]^{\gamma} .
$$

A complete design procedure includes a suitable change of coordinates to the canonical representation (2) and choice of design vectors $\ell$ and $\gamma$, but next we only sketch the proof of finite-time stability of the system in (4), as these arguments will be reused later in the distributed scenario. Compared to [12] we provide a bound for the degree of homogeneity.

\section{A. Homogeneity}

First, we check that the error system (4) is homogeneous for a proper selection of $\gamma$, meaning that $f(e)$ verifies the restrictions imposed in Definition 3 for some homogeneity degree $v<0$ and weights $\mathbf{r}$. For this goal, choose

$$
r_{s}=1+(s-1) v, \quad s=\overline{1, n},
$$

then $r_{s}>0$ for $-\frac{1}{n}<v<0$. For each $s=\overline{1, n-1}$ this gives

$$
\begin{aligned}
& f_{s}\left(\Lambda_{r}(\lambda) e\right)=\lambda^{r_{s+1}} e_{s+1}+\ell_{s} \lambda^{r_{1} \gamma_{s}}\left\lceil e_{1}\right\rfloor^{\gamma_{s}} \\
& \text { and } f_{n}\left(\Lambda_{r}(\lambda) e\right)=\ell_{n} \lambda^{r_{1} \gamma_{n}}\left\lceil e_{1}\right\rfloor^{\gamma_{n}} .
\end{aligned}
$$

Therefore, for $\gamma=\left[\begin{array}{lll}\gamma_{1} & \ldots & \gamma_{n}\end{array}\right]^{\mathrm{T}}$ in (3) with

$$
\gamma_{s}=\frac{r_{s+1}}{r_{1}}=1+s v, \quad s=\overline{1, n},
$$

the error system (4) is homogeneous with a negative degree $v$. In addition, $0<\gamma_{s}<1$.

\section{B. Stability}

Next, we show that (4) has a strictly positively invariant set. Rewrite the system (4) as follows:

$$
\dot{e}=\left(A+\ell c^{\mathrm{T}}\right) e+\operatorname{diag}(\ell)\left(\left\lceil e_{1} 1_{n}\right\rfloor^{\gamma}-e_{1} 1_{n}\right) .
$$

Let us analyze the Lyapunov function

$$
V(e)=e^{\mathrm{T}} P e,
$$

with $P=P^{\mathrm{T}}>0$ as the solution of the Lyapunov equation

$$
\tilde{A}^{\mathrm{T}} P+P \tilde{A}+Q=0,
$$

with $\tilde{A}=A+\ell c^{\mathrm{T}}$, a matrix $Q=Q^{\mathrm{T}}>0$ and under an auxiliary not restricting condition $P \geq I_{n}$.

Since the system is observable, then there always exist $\ell, P$ and $Q$ such that these linear matrix inequalities are satisfied. The time derivative of $V$ for (6) can be written as follows:

$$
\begin{aligned}
\dot{V}(e) & =-e^{\mathrm{T}} Q e+2 e^{\mathrm{T}} P \operatorname{diag}(\ell)\left(\left\lceil e_{1} 1_{n}\right\rfloor^{\gamma}-e_{1} 1_{n}\right) \\
& \leq-\frac{\lambda_{\min }(Q)}{\lambda_{\max }(P)} V+2\|e\|\|P \operatorname{diag}(\ell)\|\left\|\left\lceil e_{1} 1_{n}\right\rfloor^{\gamma}-e_{1} 1_{n}\right\|
\end{aligned}
$$

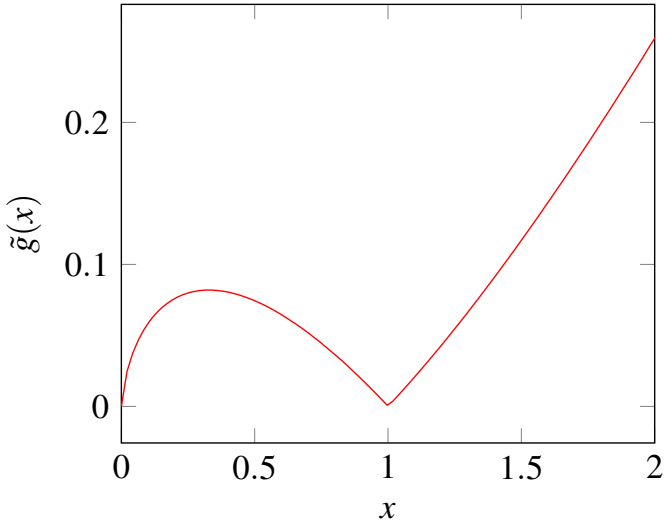

Fig. 1. Example of $\tilde{g}(x)$ with $\alpha=0.8$

Considering $e \in S=\left\{e \in \mathbb{R}^{n}: V(e)=1\right\}$ (i.e. $\|e\|^{2} \leq \lambda_{\min }^{-1}(P) \leq$ 1) we obtain

$$
\dot{V} \leq-\frac{\lambda_{\min }(Q)}{\lambda_{\max }(P)}+2\|P \operatorname{diag}(\ell)\| \sqrt{\sum_{s=1}^{n}\left(\left|e_{1}\right| \gamma_{s}-\left|e_{1}\right|\right)^{2}}
$$

Note that for $v=0$ the above expression is reduced to

$$
\dot{V} \leq-\frac{\lambda_{\min }(Q)}{\lambda_{\max }(P)} \text {. }
$$

Then by continuity it is also satisfied for a negative $v$ close to zero since $\frac{\lambda_{\min }(Q)}{\lambda_{\max }(P)}>0$.

To derive an expression for $v$, which guarantees that $(7)$ is negative definite on $S$, we have to calculate an upper bound for $\left|e_{1}\right|^{\gamma_{s}}-\left|e_{1}\right|$ taking in mind that $\left|e_{1}\right| \in[0,1]$ for $e \in S$. To get an idea, consider the function $\tilde{g}(x)=\left|x^{\alpha}-x\right|$ for $x=[0,1]$. An example of $\tilde{g}$ is given in Fig. 1 for $\alpha=0.8$. Inspired by the graph of $\tilde{g}$, we apply the Mean Value Theorem:

$$
g(a)-g(b)=g^{\prime}(\theta)(a-b), \quad \theta \in[a, b]
$$

to the function $g: \alpha \mapsto x^{\alpha}$, considering $x \in[0,1]$ as a parameter and $\alpha \in[0,1)$ as the argument. For $\alpha \in[0,1)$, we obtain

$$
g(\alpha)-g(1)=\xi(x, \theta)(\alpha-1)
$$

with $\xi(x, \theta)=\ln (x) x^{\theta}$ for some $\theta \in[\alpha, 1]$. For any such fixed $\theta, \xi(0, \theta)=\xi(1, \theta)=0$ and $\xi(x, \theta) \leq 0$ for any $x \in[0,1]$. It is easy to check that the minimal value of $\xi$ is reached at

$$
x_{\mathrm{opt}}=\exp \left(-\theta^{-1}\right), \xi\left(x_{\mathrm{opt}}, \theta\right)=-\theta^{-1} \exp (-1) .
$$

Thus, we can use the bound

$$
\left|e_{1}\right|^{\gamma_{s}}-\left|e_{1}\right| \leq \exp (-1) \frac{1-\gamma_{s}}{\gamma_{s}}=\exp (-1) \frac{-s v}{1+s v} .
$$

Additionally, using $\left|\frac{s v}{1+s v}\right| \leq\left|\frac{n v}{1+n v}\right|$ for $s=\overline{1, n}$ in 77 leads to

$$
\dot{V} \leq-\frac{\lambda_{\min }(Q)}{\lambda_{\max }(P)}+2 \exp (-1)\|P \operatorname{diag}(\ell)\| \sqrt{n} \frac{n|v|}{1+n v} .
$$

Now a bound for $v$ can be selected as

$$
|v|<\frac{\eta}{n(\sqrt{n}+\eta)}, \quad \eta=\frac{\exp (1)}{2} \frac{\lambda_{\min }(Q)}{\lambda_{\max }(P)\|P \operatorname{diag}(\ell)\|},
$$

which ensures that $\dot{V}(e)<0$ for $e \in S$ and that the set $S$ is strictly positively invariant for (4). Thus by Theorem 11 the system is globally asymptotically stable, and since $v<0$, it is globally finite-time stable by Theorem 2 


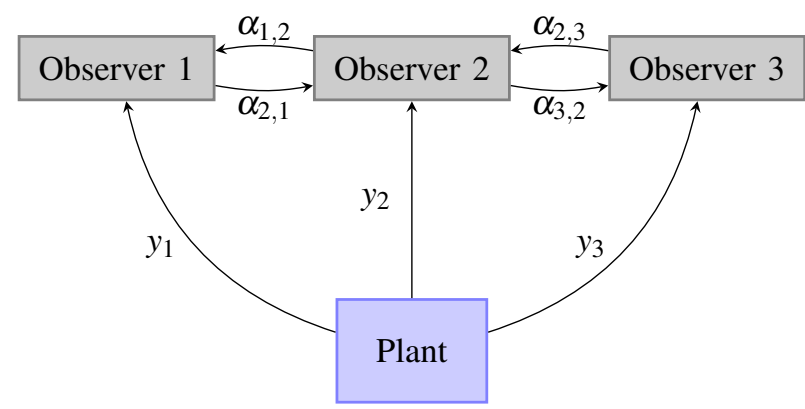

Fig. 2. Scheme depicting a distributed observer with $N=3$

\section{Distributed OBSERVER}

Now we turn to the networked observer case. In this setting, the plant has $N>1$ outputs available for measurements:

$$
\dot{x}=A x+d, \quad y_{i}=c_{i}^{\mathrm{T}} x+w_{i}, \quad i=\overline{1, N},
$$

each having an output vector $c_{i} \in \mathbb{R}^{n}$ with measurement noise $w_{i} \in \mathbb{R}$ and with an additive disturbance $d \in \mathbb{R}^{n}$ (an exogenous input or a function of the state $d=\delta(x)$ ). For $d=0$, the distributed $N$ observers are selected in the following form:

$$
\dot{z}_{i}=A z_{i}+\operatorname{diag}\left(\ell_{i}\right)\left\lceil\left(c_{i}^{\mathrm{T}} z_{i}-y_{i}\right) 1_{n}\right]^{\gamma_{i}}+\sum_{j \in \mathcal{N}_{i}} \alpha_{i, j}\left[z_{j}-z_{i}\right\rfloor^{\beta},
$$

for each $i=\overline{1, N}$, where $z_{i} \in \mathbb{R}^{n}$ is an estimate of the state generated by the $i^{\text {th }}$ observer; $\ell_{i} \in \mathbb{R}^{n}, \alpha_{i, j} \in \mathbb{R}_{+}, \gamma_{i} \in \mathbb{R}^{n}$ and $\beta \in \mathbb{R}^{n}$ are observer parameters selected later. The observers are connected in a graph and $\mathcal{N}_{i}$ denotes the set of neighboring nodes, i.e. $j \in \mathcal{N}_{i}$ if there is a directed edge from $j$ to $i\left(\alpha_{i, j}=0\right.$ otherwise), see an example in Fig. 2 with $\mathcal{N}_{1}=\{2\}, \mathcal{N}_{3}=\{2\}$ and $\mathcal{N}_{2}=\{1,3\}$. Of course, if there exists one $c_{i}$ such that the system 8 is observable from that output, then a distributed structure may become redundant, but if the pair $\left(A,\left[\begin{array}{lll}c_{1} & \ldots & c_{N}\end{array}\right]\right)$ is observable and each separate pair $\left(A, c_{i}\right)$ is not, then coupling $\alpha_{i, j} \neq 0$ between observers is obligatory.

For each node, the observer error is

$$
e_{i}=z_{i}-x
$$

and the error system is (with $d=0$ and $w_{i}=0$ )

$$
\dot{e}_{i}=A e_{i}+\operatorname{diag}\left(\ell_{i}\right)\left[1_{n}\left(c_{i}^{\mathrm{T}} e_{i}\right)\right\rfloor^{\gamma_{i}}+\sum_{j \in \mathcal{N}_{i}} \alpha_{i, j}\left[\left(e_{j}-e_{i}\right)\right\rfloor^{\beta} .
$$

The goal of this section is to propose conditions under which the interconnected systems in (11) are finite-time stable. The construction below will follow the main ideas given in Section III for the design and the analysis of a finite-time centralized observer (3).

Similar to the centralized case we presume that the plant (8) is in the form of multiple chains of integrators:

$$
\begin{aligned}
& A=\left[\begin{array}{cccc}
A_{1} & 0 & \ldots & 0 \\
0 & A_{2} & \ldots & 0 \\
& & \ddots & \\
0 & 0 & \ldots & A_{N}
\end{array}\right],
\end{aligned}
$$

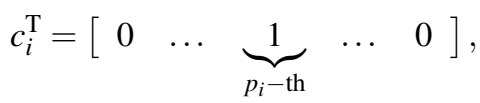

where $p_{i} \in[1, n]$ for $i=\overline{1, N}$ is such that the head of each integrator chain $A_{i} \in \mathbb{R}^{n_{i} \times n_{i}}$, with $n=\sum_{i=1}^{N} n_{i}$, is measured by a single observer node. Since each node can only reconstruct the partial state corresponding to its integrator chain (i.e. the pairs $\left(A_{i}, c_{i}\right)$ are observable, but not $\left.\left(A, c_{i}\right)\right)$ the observers must be connected in a network.

Unlike in the centralized case, there does not always exist a coordinate transformation to the form (12) and (13), hence it does not constitute a canonical form for the distributed estimation problem, especially since the integrators are disconnected. However, in Section IV-C we show that if the observed system is not in the form of (12), the stability result is still valid under some conditions.

\section{A. Homogeneity}

Lemma 1: The system (11) with (12), (13) and $d=0, w_{i}=0$ is homogeneous with respect to the weights in (5) for

$$
\gamma_{i, s}=\frac{1+s v}{1+\left(p_{i}-1\right) v}, \quad \beta_{s}=\frac{1+s v}{1+(s-1) v},
$$

where $i=\overline{1, N}$ and $s=\overline{1, n}$.

Note that the properties $0<\beta_{s}<1$ and $\gamma_{i, s}>0$ are satisfied by construction.

Proof: For $s=\overline{1, n-1}$ the relation

$$
\begin{aligned}
& \lambda^{v} \lambda^{1+(s-1) v} \dot{e}_{i, s}=\lambda^{1+s v} \mu_{s} e_{i, s+1}-\ell_{i, s} \lambda^{\left(1+\left(p_{i}-1\right) v\right) \gamma_{i, s}}\left\lceil e_{p_{i}, s}\right\rfloor^{\gamma_{i, s}} \\
& +\sum_{j \in \mathcal{N}_{i}} \alpha_{i, j} \lambda^{(1+(s-1) v) \beta_{s}}\left\lceil\left(e_{j, s}-e_{i, s}\right)\right\rfloor^{\beta_{s}}
\end{aligned}
$$

has to be fulfilled, where $\mu_{s}=\left\{\begin{array}{l}0 \text { if } s \in\left\{\sum_{j=1}^{k} n_{j} \mid k=\overline{1, N}\right\} \\ 1 \text { otherwise. }\end{array}\right.$

\section{$B$. The main result formulation}

For $v=0$, the observers in 9 are standard linear Luenberger observers with couplings and the asymptotic stability of the network can be evaluated using the error system

$$
\dot{E}=W E
$$

for the large scale error $E^{\mathrm{T}}=\left[\begin{array}{lll}e_{1}^{\mathrm{T}} & \ldots & e_{N}^{\mathrm{T}}\end{array}\right] \in \mathbb{R}^{N n}$ with

$$
\begin{aligned}
& W=\operatorname{diag}\left\{A+\ell_{i} c_{i}^{\mathrm{T}}\right\}_{i=1}^{N}+H \otimes I_{n}, \\
& H=\left[h_{i j}\right]_{i, j=1}^{N}, \quad h_{i j}= \begin{cases}-\sum_{k \in \mathcal{N}_{i}} \alpha_{i, k} & \text { for } i=j, \\
\alpha_{i, j} & \text { for } i \neq j .\end{cases}
\end{aligned}
$$

We now assume that with $\gamma_{i}=1_{n}, \beta=1_{n}$ and appropriate gains $l_{i}, \alpha_{i, j}$ in (9) an asymptotically converging observer can be designed (possible using methods referenced in the introduction). This justifies the following assumption:

Assumption 1: There exist solutions $P=P^{\mathrm{T}}>0$ and $Q=$ $Q^{\mathrm{T}}>0$ of the following system of linear matrix inequalities:

$$
W^{\mathrm{T}} P+P W+Q=0, \quad P \geq I_{N n} .
$$

Now we may formulate the main result of this note:

Theorem 4: Let Assumption 1 be satisfied and $d=0, w_{i}=0$, then the estimation error of the observer network (9) with (8), 
(12), (13) is globally finite-time stable, provided that $\gamma_{i}, \beta$ are chosen according to (14) and

$$
|v| \leq \frac{(2 n-1) h+a+b-\sqrt{(h+a+b)^{2}-4 b h}}{2((a+n h)(n-1)+b n)},
$$

where

$$
\begin{aligned}
a & =\left\|P \operatorname{diag}\left\{\operatorname{diag}\left(\ell_{i}\right)\right\}_{i=1}^{N}\right\| \exp (-1) n \sqrt{N n}, \\
b & =\|P M\| \ln (2) 2 N \sqrt{n}, \quad h=\frac{\lambda_{\min }(Q)}{2 \lambda_{\max }(P)}, \\
M & =\operatorname{diag}\left\{\alpha_{i}^{\mathrm{T}} \otimes I_{n}\right\}_{i=1}^{N}, \quad \alpha_{i}^{\mathrm{T}}=\left[\begin{array}{lll}
\alpha_{i 1} & \ldots & \alpha_{i N}
\end{array}\right],
\end{aligned}
$$

and $P, Q$ are the solutions of $(16)$.

The proof is given in Subsection VII

Remark 1: The inequality (17) always admits a solution and consequently, the choice of $v$ depend on $\ell_{i}$ and $\alpha_{i, j}$, and it will influence the settling time: it is expected to be smaller for a decreasing degree of homogeneity. It is obtained by replacing in (26) and 277) (see below) the variables $s$ and $p_{i}$ by their limit values 1 and $n$, which is a rather conservative substitution allowing a guaranteed upper bound to be derived explicitly. Then, for an application, the left-hand side of expressions 26 and (27) can be used.

Remark 2: A fixed-time distributed observer might be designed by introducing additional terms with positive homogeneity degree in 99:

$\dot{z}_{i}=A z_{i}+\operatorname{diag}\left(\ell_{i}\right) \sum_{k=1}^{2}\left\lceil\left(c_{i}^{\mathrm{T}} z_{i}\right) 1_{n}-y_{i} 1_{n}\right\rfloor^{\gamma_{i}^{k}}+\sum_{j \in \mathcal{N}_{i}} \alpha_{i, j} \sum_{k=1}^{2}\left\lceil z_{j}-z_{i}\right\rfloor^{\beta^{k}}$,

where, following (14):

$$
\gamma_{i, s}^{k}=\frac{1+s v_{k}}{1+\left(p_{i}-1\right) v_{k}}, \quad \beta_{s}^{k}=\frac{1+s v_{k}}{1+(s-1) v_{k}}
$$

for $i=\overline{1, N}, s=\overline{1, n}$ with $-\frac{1}{n}<v_{1}<0$ and $v_{2}>0$. The proof of fixed-time stability of this observer would follow local homogeneity arguments (as in [10], [30]) and by repeating the main steps used in the proof of Theorem 4

\section{Robustness with respect to disturbances}

We turn to the case where the plant is disturbed by $d \neq 0$ and the measurements are tainted by noise $w_{i} \neq 0$ (e.g. due to sensor imperfections), which for $i=\overline{1, N}$ yields the error system

$\dot{e}_{i}=A e_{i}+\operatorname{diag}\left(\ell_{i}\right)\left[1_{n}\left(c_{i}^{\mathrm{T}} e_{i}-w_{i}\right)\right]^{\gamma_{i}}+\sum_{j \in \mathcal{N}_{i}} \alpha_{i, j}\left[\left(e_{j}-e_{i}\right)\right]^{\beta}-d$.

Corollary 1: Let the networked observer $(9)$ with $(8),(12)$, (13) satisfy the conditions of Theorem 4 , then its error system (18) is ISS with respect to $d$ and $w_{i}$.

Proof: Recall that the weights $\mathbf{r}$ in Lemma 1 take the form $r_{s}=1+(s-1) v, s=\overline{1, n}$. The proper weights for $d$ and $w_{i}$ for homogeneity in the sense of Theorem 3 are $\tilde{\mathbf{r}}_{d}=\mathbf{r}+1_{n} v$, $\tilde{\mathbf{r}}_{w_{i}}=r_{p_{i}}$ respectively, as can be seen from the equation

$$
\begin{gathered}
\lambda^{v} \lambda^{1+(s-1) v} \dot{e}_{i, s}=\lambda^{1+s v} \mu_{s} e_{i, s+1}-\ell_{i, s} \lambda^{\left(1+\left(p_{i}-1\right) v\right) \gamma_{i, s}}\left\lceil e_{p_{i}, s}-w_{i}\right]^{\gamma_{i, s}} \\
+\sum_{j \in \mathcal{N}_{i}} \alpha_{i, j} \lambda^{(1+(s-1) v) \beta_{s}}\left\lceil e_{j, s}-e_{i, s}\right]^{\beta_{s}}+\lambda^{v} \lambda^{1+(s-1) v} d
\end{gathered}
$$

for $s=\overline{1, n-1}$ and $\mu_{s} \in\{0,1\}$. The global asymptotic stability of the error system for $d=0, w_{i}=0$ follows from the global finite-time stability of (11).

It follows that due to its homogeneity, the distributed observer is robust with respect to disturbances of the plant and measurement noises. Note that with a similar reasoning, the network is also robust to noise in the communication channel.

Moreover, using the features of ISS, the finite-time stability is retained for an additional state feedback provided it is bounded by a nonlinear stability margin [20]. For a homogeneous system of negative degree a stability margin can be estimated to be

$$
\rho\left(\|e\|_{r}\right)=\kappa \min \left\{\|e\|_{r}^{\tilde{r}_{\max }},\|e\|_{r}^{\tilde{r}_{\min }}\right\},
$$

with $\tilde{\mathbf{r}}$ from Theorem 3 and a constant $\kappa>0$ [27]. Using this and Corollary 1, we can extend the results of Theorem 4 to a more general class of systems.

Corollary 2: Assume that all conditions of Theorem 4 are satisfied for the plant (8) with (12), (13) and $d=\delta(x)$, where $\delta: \mathbb{R}^{n} \mapsto \mathbb{R}^{n}$ is a locally Lipschitz continuous function modelling more general system dynamics. Then the estimation error of the observer network

$\dot{z}_{i}=A z_{i}+\operatorname{diag}\left(\ell_{i}\right)\left[\left(c_{i}^{\mathrm{T}} z_{i}-y_{i}\right) 1_{n}\right\rfloor^{\gamma_{i}}+\sum_{j \in \mathcal{N}_{i}} \alpha_{i, j}\left[z_{j}-z_{i}\right\rfloor^{\beta}+\delta\left(z_{i}\right)$ is globally finite-time stable if

$$
\left\|\delta\left(z_{i}\right)-\delta(x)\right\| \leq \rho\left(\left\|z_{i}-x\right\|_{r}\right),
$$

where $\rho$ is defined in $(19)$ with $\tilde{\mathbf{r}}=\tilde{\mathbf{r}}_{d}$ from Corollary 1

Compared to (11), the resulting error system will have an additional term $\delta\left(z_{i}\right)-\delta(x)$ and for a general linear system $\delta(x)=\Delta x$ with $\Delta \in \mathbb{R}^{n \times n}$, it takes the form $\Delta\left(z_{i}-x\right)=\Delta e_{i}$. Then if the bound 20) is satisfied only for big or small estimation errors, practical or local stability and convergence can be ensured for the distributed observer.

\section{Boundedness with respect to known transmission delays}

Next to robustness with respect to disturbances and noises, robustness with regard to time-delay effects is a desirable property of distributed observers, when communication delays are induced by the network.

In [31], the concept of homogeneity was defined analogously to Definition 3 for time-delayed systems described by functional differential equations of the retarded type

$$
\dot{x}(t)=f\left(x_{t}\right), \quad t \geq 0,
$$

where $x(t) \in \mathbb{R}^{n}$ and $x_{t} \in \mathfrak{C}_{[-\tau, 0]}$ is the state function, $x_{t}(s)=$ $x(t+s),-\tau \leq s \leq 0$ and $f: \mathcal{C}_{[-\tau, 0]} \rightarrow \mathbb{R}^{n}$. Here $\mathcal{C}_{[-\tau, 0]}$ denotes the Banach space of continuous functions $\phi:[-\tau, 0] \rightarrow \mathbb{R}^{n}$ with the uniform norm $\|\phi\|:=\sup _{-\tau \leq \xi \leq 0}|\phi(\xi)|$. In the following lemma, $\mathcal{B}_{\varepsilon}^{\tau}:=\left\{\phi \in \mathcal{C}_{[-\tau, 0]}:\|\phi\|_{r} \leq \varepsilon\right\}$.

Lemma 2 ([32]): Let $f\left(x_{t}\right)=F[x(t), x(t-\tau)]$ (i.e. it has a pointwise-delay), in (21) be uniformly continuous and the system 21 be $\mathbf{r}$-homogeneous with degree $v<0$ and globally asymptotically stable at the origin for $\tau=0$, then for any $\varepsilon>0$ there is $0<\tau_{0}<+\infty$ such that (21) is globally asymptotically stable with respect to $\mathcal{B}_{\varepsilon}^{\tau}$ for any delay $0 \leq \tau \leq \tau_{0}$. 
We now assume that there is a delay in the communication channel between the observer nodes which is known (e.g. using time stamps and a clock synchronization method [33]). Delays in the measurements are not included, especially because the distributed design allows to select a configuration which specifically avoids measurement delays.

Therefore, at node $i$ the estimates from neighboring nodes $z_{j}, j \in \mathcal{N}_{i}$ are delayed by $0 \leq \tau_{i, j} \leq \tau$. Since the $\tau_{i, j}$ are known, the delays can be countered by shifting the state of the observer $z_{i}(t)$ by the same $\tau_{i, j}$ in the cross-coupling term:

$$
\begin{aligned}
\dot{z}_{i}(t)=A z_{i}(t)+ & \operatorname{diag}\left(\ell_{i}\right)\left\lceil 1_{n}\left(c_{i}^{\mathrm{T}} z_{i}(t)-y_{i}(t)\right)\right\rfloor^{\gamma_{i}} \\
& +\sum_{j \in \mathcal{N}_{i}} \alpha_{i, j}\left\lceil z_{j}\left(t-\tau_{i, j}\right)-z_{i}\left(t-\tau_{i, j}\right)\right\rfloor^{\beta},
\end{aligned}
$$

In the undisturbed case this leads to the error system

$$
\begin{aligned}
\dot{e}_{i}(t)=A e_{i}(t)+ & \operatorname{diag}\left(\ell_{i}\right)\left[1_{n} c_{i}^{\mathrm{T}} e_{i}(t)\right]^{\gamma_{i}} \\
& +\sum_{j \in \mathcal{N}_{i}} \alpha_{i, j}\left[e_{j}\left(t-\tau_{i, j}\right)-e_{i}\left(t-\tau_{i, j}\right)\right]^{\beta}
\end{aligned}
$$

and if $\gamma_{i}$ and $\beta$ are chosen as in Lemma 1 then it is homogeneous with negative degree and Lemma 2 can be applied:

Corollary 3: Let the networked observer 22) with (8), (12), (13) satisfy the conditions of Theorem 4, then for any upper bound on the asymptotic estimation error there exists an admissible transmission delay $\tau_{i, j} \geq 0$.

\section{EXAMPLE}

Let us consider an example with a linear system of order $n=7$ and $N=3$ outputs:

$$
\dot{x}=\left[\begin{array}{ccccccc}
-1.5 & -0.9 & 0 & 0 & 0 & 0.2 & 0.2 \\
-0.5 & -0.7 & 0 & 0 & 0 & 0.1 & 0.1 \\
0 & 0 & 0 & -1.5 & 0 & 0.1 & 0.1 \\
0 & 0 & 0 & 0.1 & -1.1 & 0.1 & 0.1 \\
0 & 0 & 0 & 0 & 0 & 0.1 & 0.1 \\
0 & 0 & 0 & 0 & 0 & -0.8 & -1.0 \\
0 & 0 & 0 & 0 & 0 & -1.0 & -0.8
\end{array}\right] x,
$$

The observer nodes are connected in a graph as shown in Fig. 2 Observer 1 measures the first coordinate of the state vector $x$ (i.e. $c_{1}=\left[\begin{array}{lllllll}1 & 0 & 0 & 0 & 0 & 0 & 0\end{array}\right]^{\mathrm{T}}$ ), Observer 2 the third coordinate and Observer 3 the sixth.

First, a solution of (16) for $Q=I_{n N}$ is obtained. Using the method presented in [34], the observer gains are selected as $\alpha_{1,2}=\alpha_{2,1}=\alpha_{2,3}=\alpha_{3,2}=4$ and

$$
\begin{aligned}
\ell_{1} & =\left[\begin{array}{llllll}
-1.6 & 1.87 & 0.04 & -0.07 & 0.04 & 0
\end{array}\right]^{\mathrm{T}}, \\
\ell_{2} & =\left[\begin{array}{lllllll}
0 & 0 & -13.2 & 17.55 & -8.48 & 0 & 0
\end{array}\right]^{\mathrm{T}}, \\
\ell_{3} & =\left[\begin{array}{lllllll}
-0.62 & 0.27 & -0.11 & -0.83 & 0.93 & -2.8 & 2.48
\end{array}\right]^{\mathrm{T}} .
\end{aligned}
$$

Next, this leads with 17 to the bound $v \geq-3.8 \times 10^{-4}$.

As already stated in Remark 11, the resulting $v$ is rather conservative and the corresponding settling time is too large to show the finite time behavior visually. If compared to the linear observer (i.e. $v=0$ ) the error will reach asymptotically machine precision only slightly However, following Remark 1 a more accurate assessment of $v$ is possible. For instance, in

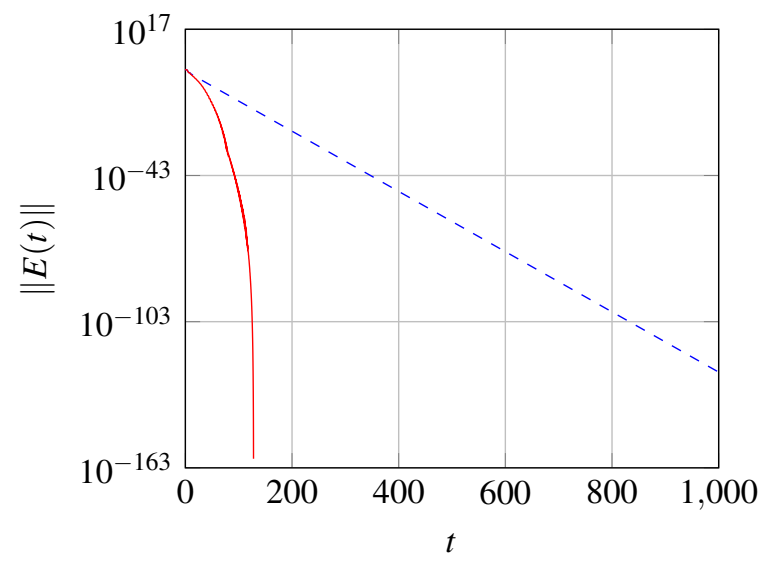

Fig. 3. Comparison of the error for the linear (dashed) and finite-time observer (solid) in logarithmic scaling.

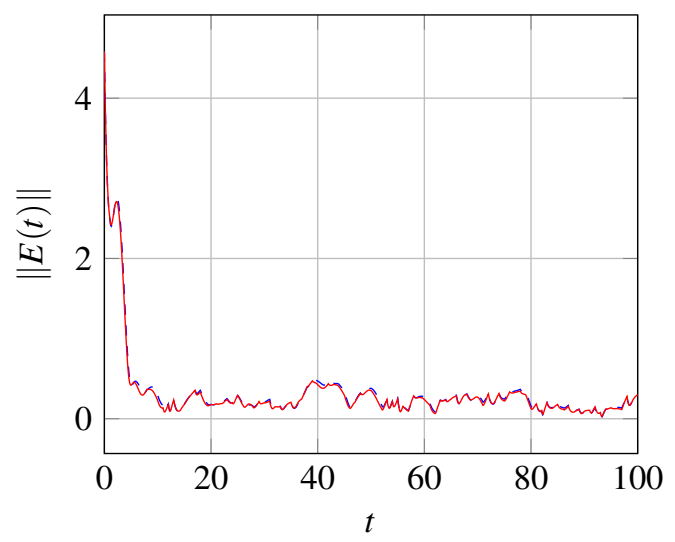

Fig. 4. Comparison of the error for the linear (dashed) and finite-time observer (solid) in case of disturbances and measurement noise.

Fig. 3 the error norm $\|E(t)\|$ is plotted in a logarithmic scale with $v=-0.02$ and compared to the linear observer with the same gains, clearly showing the finite time behavior for the former and the exponential decrease for the latter.

The error in the presence of zero-mean uniformly distributed noise and disturbance of magnitude 1 is shown in Fig. 4 for the same example. This demonstrates that the linear and finitetime observers have similar robustness performances.

Furthermore, the example is tested to illustrate Corollary 3 with a transmission delay of $\tau_{i, j}=0.27$ for all communications. The delays are compensated using observers of the form 22. As expected from the linear version of the observer its margin of stability is reached for a sufficiently big delay, and thus the error grows to infinity, see Fig. 5. In contrast, the error of the finite-time observer stays bounded, demonstrating its advantage when coping with transmission delays.

\section{CONCLUSION}

A distributed observer was designed having the ability of converging in a finite or in a fixed time. Using homogeneity concepts and properties, we have shown that the designed observer possesses the input-to-state stability property with respect to additive disturbances and measurement noises. This 


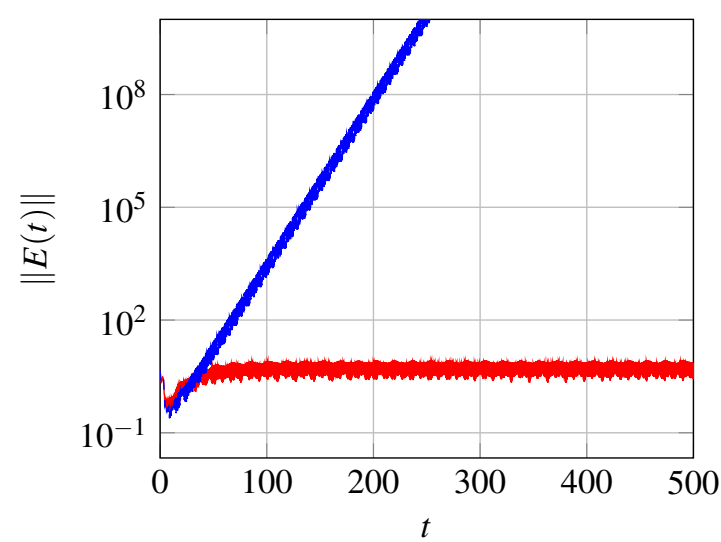

Fig. 5. Comparison of the error for the linear observer (dashed) and finitetime observer (solid) for transmission delays of $\tau_{j}=0.27$.

resulted into a class of systems for which the design can be applied, but the extension of the results to general linear systems is subject to future work. The error of the finite-time observer stays bounded for any known transmission delays which are accounted for. It would be interesting to consider varying or unknown delays. Additionally, refined estimates on the degrees of homogeneity for the fixed-time observer found in the present technical note should be investigated.

\section{Proof of Theorem 4}

The large scale error system in (11) can be rewritten as:

$$
\begin{aligned}
& \dot{E}=\operatorname{diag}\{A\}_{i=1}^{N} E \\
& +\operatorname{diag}\left\{\operatorname{diag}\left(\ell_{i}\right)\right\}_{i=1}^{N}\left\lceil\left(\operatorname{diag}\left\{c_{i}^{\mathrm{T}}\right\}_{i=1}^{N} \otimes 1_{n}\right) E\right\rfloor^{\tilde{\gamma}}+M\lceil\Gamma E\rfloor^{\tilde{\beta}},
\end{aligned}
$$

with the notation $\tilde{\gamma}^{\mathrm{T}}=\left[\begin{array}{lll}\gamma_{1}^{\mathrm{T}} & \ldots & \gamma_{N}^{\mathrm{T}}\end{array}\right], \tilde{\beta}=1_{N^{2}} \otimes \beta$ and

$$
\Gamma=\left[\begin{array}{c}
\Gamma_{1} \\
\vdots \\
\Gamma_{N}
\end{array}\right], \Gamma_{i}=\left[\begin{array}{c}
\Gamma_{i 1} \\
\vdots \\
\Gamma_{i N}
\end{array}\right], \Gamma_{i j}=[0 \ldots \underbrace{-I_{n}}_{i-\text { th }} \ldots \underbrace{I_{n}}_{j-\text { th }} \ldots 0]
$$

( $\Gamma_{i i}$ has only entries equal zero). Rewriting (24) as

$$
\begin{aligned}
\dot{E}=W E+ & \operatorname{diag}\left\{\operatorname{diag}\left(\ell_{i}\right)\right\}_{i=1}^{N}\left(\left\lceil\left(\operatorname{diag}\left\{c_{i}^{\mathrm{T}}\right\}_{i=1}^{N} \otimes 1_{n}\right) E\right\rfloor^{\tilde{\gamma}}\right. \\
& \left.-\left(\operatorname{diag}\left\{c_{i}^{\mathrm{T}}\right\}_{i=1}^{N} \otimes 1_{n}\right) E\right)+M\left(\lceil\Gamma E\rfloor^{\tilde{\beta}}-\Gamma E\right)
\end{aligned}
$$

and using the Lyapunov function $V(E)=E^{\mathrm{T}} P E$, we repeat the steps presented in Section III and for $E \in \mathcal{S}=\left\{E \in \mathbb{R}^{N n}\right.$ : $V(E)=1\}$ we obtain

$$
\begin{aligned}
& \dot{V} \leq-\frac{\lambda_{\min }(Q)}{\lambda_{\max }(P)}+2\left(\left\|P \operatorname{diag}\left\{\operatorname{diag}\left(\ell_{i}\right)\right\}_{i=1}^{N}\right\|\right. \\
& \times \sqrt{\sum_{i=1}^{N} \sum_{s=1}^{n}\left(\left|e_{p_{i}, s}\right| \gamma_{i, s}-\left|e_{p_{i}, s}\right|\right)^{2}} \\
&+\|P M\| \sqrt{\left.\sum_{i=1}^{N} \sum_{j=1}^{N} \sum_{s=1}^{n}\left(\left|e_{j, s}-e_{i, s}\right| \beta_{s}-\left|e_{j, s}-e_{i, s}\right|\right)^{2}\right)} .
\end{aligned}
$$

Again, if $v=0$ then the above inequality is reduced to

$$
\dot{V} \leq-\frac{\lambda_{\min }(Q)}{\lambda_{\max }(P)}<0
$$

Such an inequality may be preserved for a sufficiently small $v$, so let us try to estimate its value.

1) First square root: The first terms in $(25)$ are similar to the ones in (7), but $\gamma_{i, s}$ can be greater than one. Therefore, two cases have to be distinguished when applying the Mean Value Theorem:

$$
\begin{gathered}
\left|e_{p_{i}, s}\right| \gamma_{i, s}-\left|e_{p_{i}, s}\right| \leq \exp (-1) \frac{\left|1-\gamma_{i, s}\right|}{\theta} \\
\theta \in \begin{cases}{\left[\gamma_{i, s}, 1\right]} & \text { if } s>p_{i}-1, \\
{\left[1, \gamma_{i, s}\right]} & \text { if } s \leq p_{i}-1,\end{cases}
\end{gathered}
$$

which gives

$$
\frac{1-\gamma_{i, s}}{\theta} \leq \frac{1-\gamma_{i, s}}{\gamma_{i, s}}=\frac{\left(p_{i}-1-s\right) v}{1+s v}
$$

or

$$
\frac{\gamma_{i, s}-1}{\theta} \leq \gamma_{i, s}-1=\frac{\left(s-p_{i}+1\right) v}{1+\left(p_{i}-1\right) v},
$$

and subsequently for $1 \leq s, p_{i} \leq n$ :

$$
\left|\frac{\gamma_{i, s}-1}{\theta}\right| \leq \begin{cases}\left|\frac{n v}{1+n v}\right| & \text { if } s>p_{i}-1 \\ \left|\frac{(n-2) v}{1+(n-1) v}\right| & \text { if } s \leq p_{i}-1\end{cases}
$$

The right-hand side of (26) is bigger than of (27), so

$$
\sqrt{\sum_{i=1}^{N} \sum_{s=1}^{n}\left(\left|e_{p_{i}, s}\right| \gamma_{i, s}-\left|e_{p_{i}, s}\right|\right)^{2}} \leq \exp (-1) \sqrt{N n} \frac{n|v|}{1+n v} .
$$

2) Second square root: If $\|E\| \leq 1$, then $\left|e_{j, s}-e_{i, s}\right| \leq 2$. For a fixed $\alpha \in[0,1)$ consider the function $\tilde{g}(x)=\left|x^{\alpha}-x\right|$ for $x \in[0,2]$. It is easy to check that $\tilde{g}(0)=\tilde{g}(1)=0$, then $\tilde{g}(x)=x^{\alpha}-x$ for $x \in[0,1]$ and the maximal value of $\tilde{g}$ on this interval is reached for $\tilde{x}_{\mathrm{opt}}=\alpha^{\frac{1}{1-\alpha}}$ with $\sup _{0 \leq x \leq 1} \tilde{g}(x)=$ $\alpha^{\frac{\alpha}{1-\alpha}}-\alpha^{\frac{1}{1-\alpha}}$. For $x \in[1,2]$, a simple analysis of $\frac{\overline{\mathrm{d}} \tilde{g}(x)}{\mathrm{d} x}$ shows that $\tilde{g}$ is a strictly increasing function, then $\sup _{1 \leq x \leq 2} \tilde{g}(x)=$ $2-2^{\alpha}$. For $\alpha \in[0,1)$, the second derivatives with respect to $\alpha$ of $\alpha^{\frac{\alpha}{1-\alpha}}-\alpha^{\frac{1}{1-\alpha}}$ and $2-2^{\alpha}$ are

$$
\begin{aligned}
\frac{\mathrm{d}^{2}}{\mathrm{~d} \alpha^{2}} \alpha^{\frac{1}{1-\alpha}} & \left(\alpha^{-1}-1\right) \\
= & -\frac{\alpha^{-1 /(\alpha-1)-2}\left((1-\alpha)(\alpha-1)^{2}+\alpha(\ln (\alpha)-(\alpha-1))^{2}\right)}{(\alpha-1)^{3}}>0
\end{aligned}
$$

which means it is convex, and $\frac{\mathrm{d}^{2}}{\mathrm{~d} \alpha^{2}}\left(2-2^{\alpha}\right)=-2^{\alpha} \ln ^{2}(2)<0$, which means it is concave, respectively. Since both functions meet at $\alpha=0$ and $\alpha=1$ (see Fig. 6), we obtain:

$$
\alpha^{\frac{\alpha}{1-\alpha}}-\alpha^{\frac{1}{1-\alpha}} \leq 2-2^{\alpha} \text {. }
$$

Consequently, returning to the second square root in 25:

$$
\begin{gathered}
|| e_{j, s}-\left.e_{i, s}\right|^{\beta_{s}}-\left|e_{j, s}-e_{i, s}\right| \mid \leq 2-2^{\beta_{s}} \\
=\ln (2) 2^{\theta}\left(1-\beta_{s}\right) \leq \ln (2) 2\left(1-\beta_{s}\right)=\ln (2) 2 \frac{-v}{1+(n-1) v}
\end{gathered}
$$




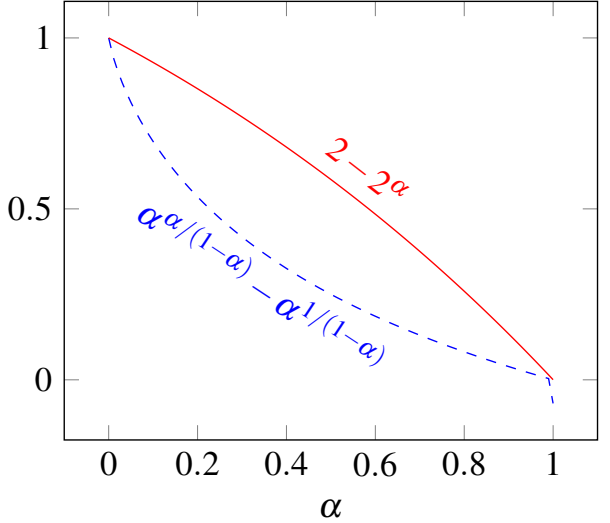

Fig. 6. Comparison of maximums for different $\alpha$

for $s=\overline{1, n}$, where $\theta \in\left[\beta_{s}, 1\right]$ and the Mean Value Theorem has been used. Therefore, we get

$$
\begin{aligned}
& \sqrt{\sum_{i=1}^{N} \sum_{j=1}^{N} \sum_{s=1}^{n}\left(\left|e_{j, s}-e_{i, s}\right| \beta_{s}-\left|e_{j, s}-e_{i, s}\right|\right)^{2}} \\
& \leq \ln (2) 2 N \sqrt{n} \frac{|v|}{1+(n-1) v}
\end{aligned}
$$

3) Combining the estimates: Inserting (28, 29) into 25]

$$
\begin{aligned}
& \dot{V} \leq-\frac{\lambda_{\min }(Q)}{\lambda_{\max }(P)}+2\left(\left\|P \operatorname{diag}\left\{\operatorname{diag}\left(\ell_{i}\right)\right\}_{i=1}^{N}\right\|\right. \\
& \left.\times \exp (-1) \sqrt{N n} \frac{n|v|}{1+n v}+\|P M\| \ln (2) 2 N \sqrt{n} \frac{|v|}{1+(n-1) v}\right) .
\end{aligned}
$$

That gives finally a quadratic equation in $|v|$, whose solution is provided in the formulation (17) of Theorem 4

\section{ACKNOWLEDGMENT}

This work has been partially supported by the project UCoCoS, funded by the European Union's Horizon 2020 research and innovation program under the Marie SkłodowskaCurie Grant Agreement No 675080. It was also partially funded by Russian Science Foundation (Project 17-19-01422), the Government of Russian Federation (Grant 074-U01) and the Ministry of Education and Science of Russian Federation (Project 14.Z50.31.0031).

\section{REFERENCES}

[1] S. Park and N. C. Martins, "Design of distributed LTI observers for state omniscience," IEEE Transactions on Automatic Control, vol. 62 , no. 2, pp. 561-576, Feb. 2017.

[2] V. Ugrinovskii, "Conditions for detectability in distributed consensusbased observer networks," IEEE Transactions on Automatic Control, vol. 58, no. 10, pp. 2659-2664, Oct. 2013.

[3] S. Park and N. C. Martins, "Necessary and sufficient conditions for the stabilizability of a class of LTI distributed observers," in 2012 IEEE 51st IEEE Conference on Decision and Control (CDC), Dec. 2012, pp. 7431-7436.

[4] U. A. Khan, S. Kar, A. Jadbabaie, and J. M. F. Moura, "On connectivity, observability, and stability in distributed estimation," in 49th IEEE Conference on Decision and Control (CDC), Dec. 2010, pp. 6639-6644.
[5] T. Kim, H. Shim, and D. D. Cho, "Distributed Luenberger observer design," in 2016 IEEE 55th Conference on Decision and Control (CDC), Dec. 2016, pp. 6928-6933.

[6] R. Olfati-Saber, "Distributed Kalman filtering for sensor networks," in 2007 46th IEEE Conference on Decision and Control, Dec. 2007, pp. 5492-5498.

[7] M. Farina, G. Ferrari-Trecate, and R. Scattolini, "Distributed moving horizon estimation for linear constrained systems," IEEE Transactions on Automatic Control, vol. 55, no. 11, pp. 2462-2475, Nov. 2010.

[8] V. Ugrinovskii, "Distributed robust filtering with consensus of estimates," Automatica, vol. 47, no. 1, pp. 1-13, 2011.

[9] L. Wang and A. S. Morse, "A distributed observer for a time-invariant linear system," IEEE Transactions on Automatic Control, 2017.

[10] V. Andrieu, L. Praly, and A. Astolfi, "Homogeneous approximation, recursive observer design, and output feedback," SIAM J. Control Optimization, vol. 47, no. 4, pp. 1814-1850, 2008.

[11] A. Levant, "Homogeneity approach to high-order sliding mode design," Automatica, vol. 41, no. 5, pp. 823-830, 2005.

[12] W. Perruquetti, T. Floquet, and E. Moulay, "Finite-time observers: Application to secure communication," IEEE Transactions on Automatic Control, vol. 53, no. 1, pp. 356-360, Feb. 2008.

[13] H. Ríos and A. Teel, "A hybrid observer for uniform finite-time state estimation of linear systems," in In Proc. 55th IEEE Conference on Decision and Control (CDC), Las Vegas, 2016.

[14] F. Lopez-Ramirez, D. Efimov, A. Polyakov, and W. Perruquetti, "Fixed-time output stabilization of a chain of integrators," in 2016 IEEE 55th Conference on Decision and Control (CDC), Dec. 2016, pp. 3886-3891.

[15] S. P. Bhat and D. S. Bernstein, "Geometric homogeneity with applications to finite-time stability," Mathematics of Control, Signals and Systems, vol. 17, pp. 101-127, 2005.

[16] H. Silm, R. Ushirobira, D. Efimov, J. P. Richard, and W. Michiels, "A distributed finite-time observer for linear systems," in 2017 IEEE 56th Annual Conference on Decision and Control (CDC), Dec. 2017, pp. 1731-1736.

[17] E. Roxin, "On finite stability in control systems," Rendiconti del Circolo Matematico di Palermo, vol. 15, pp. 273-283, 1966.

[18] H. K. Khalil, Nonlinear Systems, ser. NJ 07458. Upper Saddle River: Prentice-Hall, 1996.

[19] A. Polyakov, "Nonlinear feedback design for fixed-time stabilization of linear control systems," IEEE Transactions on Automatic Control, vol. 57, no. 8, pp. 2106-2110, 2012.

[20] S. N. Dashkovskiy, D. V. Efimov, and E. D. Sontag, "Input to state stability and allied system properties," Automation and Remote Control, vol. 72, no. 8, p. 1579, 2011.

[21] V. Zubov, "On systems of ordinary differential equations with generalized homogenous right-hand sides," Izvestia vuzov. Mathematica., vol. 1, pp. 80-88, 1958 (in Russian).

[22] A. Bacciotti and L. Rosier, Lyapunov Functions and Stability in Control Theory, 2nd. Springer, Berlin, 2005.

[23] L. Rosier, "Homogeneous Lyapunov function for homogeneous continuous vector field," Systems \& Control Letters, vol. 19, pp. 467-473, 1992.

[24] S. P. Bhat and D. S. Bernstein, "Finite time stability of continuous autonomous systems," SIAM Journal on Control and Optimization, vol. 38, no. 3, pp. 751-766, 2000.

[25] E. Bernuau, A. Polyakov, D. Efimov, and W. Perruquetti, "On extension of homogeneity notion for differential inclusions," in Proceeding of the European Control Conference, Zurich, 2013, pp. 2204-2209.

[26] H. Nakamura, Y. Yamashita, and H. Nishitani, "Smooth Lyapunov functions for homogeneous differential inclusions," in Proceedings of the 41st SICE Annual Conference., vol. 3, Aug. 2002, pp. 1974-1979.

[27] E. Bernuau, A. Polyakov, D. Efimov, and W. Perruquetti, "Verification of ISS, iISS and IOSS properties applying weighted homogeneity," Systems \& Control Letters, vol. 62, pp. 1159-1167, 2013.

[28] A. J. Krener and A. Isidori, "Linearization by output injection and nonlinear observers," Systems \& Control Letters, vol. 3, no. 1, pp. 4752, 1983.

[29] A. J. Krener and W. Respondek, "Nonlinear observers with linearizable error dynamics," SIAM Journal on Control and Optimization, vol. 23, no. 2, pp. 197-216, 1985.

[30] D. Efimov and W. Perruquetti, "On conditions of oscillations and multihomogeneity," Mathematics of Control, Signals, and Systems, vol. 28, no. 1, pp. 1-37, 2015.

[31] - "Homogeneity for time-delay systems," IFAC Proceedings Volumes, vol. 44, no. 1, pp. 3861-3866, 2011. 
[32] K. Zimenko, D. Efimov, A. Polyakov, and W. Perruquetti, "A note on delay robustness for homogeneous systems with negative degree," Automatica, vol. 79, pp. 178-184, 2017.

[33] A. Kruszewski, W. Jiang, E. Fridman, J. P. Richard, and A. Toguyeni, "A switched system approach to exponential stabilization through communication network," IEEE Transactions on Control Systems Technology, vol. 20, no. 4, pp. 887-900, Jul. 2012.

[34] H. Zhu, K. Liu, J. L'u, Z. Lin, and Y. Chen, "On the cooperative observability of a continuous-time linear system on an undirected network," in 2014 International Joint Conference on Neural Networks (IJCNN), Jul. 2014, pp. 2940-2944. 